\title{
Effect of non-adiabatic coupling on the isotopic dependence of the photodissociation cross section of $\mathrm{CO}$
}

\author{
L. Andric ${ }^{1}$, F. Bouakline ${ }^{1}$, T. P. Grozdanov ${ }^{2}$, and R. McCarroll ${ }^{1}$ \\ ${ }^{1}$ Laboratoire de Chimie Physique-Matière et Rayonnement (UMR 7614 du CNRS), Université Pierre et Marie Curie, \\ 75231 Paris Cedex 05, France \\ 2 Institute of Physics, PO Box 57, 11001-Belgrade, Serbia and Montenegro
}

Received 16 December 2003 / Accepted 15 March 2004

\begin{abstract}
Calculations of the photodissociation cross sections of $\mathrm{CO}$ from the ground $\mathrm{X}^{1} \Sigma^{+}$state into the coupled Rydberg $\mathrm{B}^{1} \Sigma^{+}$state and the valence dissociative $\mathrm{D}^{\prime 1} \Sigma^{+}$state have been carried out using the smooth exterior complex scaling (SECS) method. Results are presented for all of the 6 isotopomers involving the ${ }^{12} \mathrm{C},{ }^{13} \mathrm{C},{ }^{16} \mathrm{O},{ }^{17} \mathrm{O}$ and ${ }^{18} \mathrm{O}$ isotopes. The absorption profiles of the predissociating resonances induced by non-adiabatic coupling vary considerably from one isotopomer to another. It is confirmed that resonance widths obtained simply from a diagonalization of the complex Hamiltonian do not give a true indication of the absorption profile. A correct profile is only obtained when the sum over all resonant and non-resonant continuum states is performed. A brief discussion is given of the implications for isotopic fractionation in interstellar CO.
\end{abstract}

Key words. astrochemistry - ISM: molecules - molecular processes - ultraviolet: ISM

\section{Introduction}

Carbon monoxide, the second most abundant interstellar molecule after $\mathrm{H}_{2}$, has been extensively observed in a large number of astronomical objects over a wide spectral range covering both electronic transitions in the vacuum ultra-violet (Smith et al. 1991; Sheffer et al. 1992, 2002) and rotational transitions in the millimetre-wave band (Penzias et al. 1972; Mahoney et al. 1976; Dickman et al. 1977; Langer et al. 1980). But what makes CO of particular interest for the diagnostics of the interstellar clouds is the possibility of observing the other isotopomers: not only ${ }^{12} \mathrm{C}^{16} \mathrm{O}$ and the fairly common ${ }^{13} \mathrm{C}^{16} \mathrm{O}$ (Penzias et al. 1972), but also the rarer species ${ }^{12} \mathrm{C}^{18} \mathrm{O}$ (Mahoney et al. 1976), ${ }^{12} \mathrm{C}^{17} \mathrm{O}$ (Dickman et al. 1977), ${ }^{13} \mathrm{C}^{18} \mathrm{O}$ (Langer et al. 1980) and, more recently, even the rarest of them all ${ }^{13} \mathrm{C}^{17} \mathrm{O}$ (Bensch et al. 2001).

The observations show that the relative abundance of the different isotopomers seems to vary from one object to another, thereby indicating the existence of a non-thermal isotopic fractionation of the $\mathrm{C}, \mathrm{O}$ isotopes in the $\mathrm{CO}$ molecule. But while there is general agreement on the main physical mechanisms leading to fractionation in CO (van Dishoeck \& Black 1988), we are still some way from extracting with certainty the ${ }^{12} \mathrm{C} /{ }^{13} \mathrm{C}$ or ${ }^{16} \mathrm{O} /{ }^{17} \mathrm{O} /{ }^{18} \mathrm{O}$ isotopic abundances, from spectral observations of $\mathrm{CO}$ alone. In addition, the analysis of the fine structure $\left({ }^{2} \mathrm{P}_{1 / 2} \rightarrow{ }^{2} \mathrm{P}_{3 / 2}\right)$ transitions in ${ }^{12} \mathrm{C}^{+}$and ${ }^{13} \mathrm{C}^{+}$(Boreiko $\&$ Betz 1996) and the $\left({ }^{3} \mathrm{P}_{2} \rightarrow{ }^{3} \mathrm{P}_{1}\right)$ transitions in neutral ${ }^{12} \mathrm{C}$

Send offprint requests to: R. McCarroll, e-mail: mccarrol@ccr.jussieu.fr and ${ }^{13} \mathrm{C}$ (Keene et al. 1998) seems to suggest that even when there is isotopic fractionation in $\mathrm{CO}$, the abundance of free ${ }^{13} \mathrm{C}$ and ${ }^{13} \mathrm{C}^{+}$does not seem to be significantly affected.

It is generally assumed (van Dishoeck \& Black 1988) that there are two main processes which are responsible for nonthermal fractionation of the $\mathrm{CO}$ isotopes: one, collisional the other radiative, which lead to abundance ratios of the $\mathrm{CO}$ isotopomers, different from those expected from the local abundances of the different isotopes of the $\mathrm{C}$ and $\mathrm{O}$ nuclei. The principal collisional process involves the exoenergic ion-molecule reaction leading to isotope exchange (Watson et al. 1976):

${ }^{13} \mathrm{C}^{+}+{ }^{12} \mathrm{C}^{16} \mathrm{O} \rightarrow{ }^{13} \mathrm{C}^{16} \mathrm{O}+{ }^{12} \mathrm{C}^{+}+\Delta E(=34.6 \mathrm{~K})$

which tends to enrich the ${ }^{13} \mathrm{C}$-containing isotopomers of $\mathrm{CO}$ in low temperature clouds. It would seem (van Dishoeck \& Black 1988) that reaction (1) only contributes to isotopic fractionation at low temperatures (of the order of $50 \mathrm{~K}$ or less). At higher temperatures, the inverse endothermic reaction will tend to diminish the net isotopic fractionation. The analogous reaction with $\mathrm{O}^{+}$ions is of little importance simply because there are few $\mathrm{O}^{+}$ions in the medium (the interstellar photons having insufficient energy to ionize neutral $\mathrm{O}$ atoms).

The radiative process involving photodissociation affects all the $\mathrm{CO}$ isotopomers in a selective manner. In the range of photon energies present in the interstellar environment, photodissociation occurs primarily via predissociating resonances of the $\mathrm{CO}$ molecule and as a consequence, the photodissociation cross sections are quite frequency selective and only photons in narrow frequency bands are capable of dissociating CO. 
But, correspondingly, photons in these bands are strongly absorbed as they penetrate a cloud and a self shielding of $\mathrm{CO}$ occurs (just as for the $\mathrm{H}_{2}$ molecule which is also dissociated via a resonance). The amount of self shielding depends on many factors (interstellar radiation field, the cloud density, etc.) but if it can be assumed that the resonances of different isotopomers do not overlap, then the rarer isotopomers will be subject to less self shielding than the common ${ }^{12} \mathrm{C}^{16} \mathrm{O}$. This leads to a non thermal fractionation of the various isotopic forms of $\mathrm{CO}$.

However, the assumption of non-overlapping absorption profiles of the various isotopomers depends on the nature of the predissociating resonances. Our recent calculations (Andric et al. 1999) on the influence of resonances on the photoabsorption cross sections of $\mathrm{CO}$ in the photon energy range [90000, $106000 \mathrm{~cm}^{-1}$ did indeed show that the lower lying resonances (shape resonances due to tunnelling through a potential barrier) are sufficiently narrow so that no overlap of the resonances of different isotopomers may be expected. But for the higher resonances, which are of the Feshbach type (due to non adiabatic coupling between a discrete and the dissociating continuum state), most of the absorption profiles are much wider and a considerable overlap of the resonances of different isotopomers may be expected. It therefore seemed of interest to perform a systematic investigation of all the isotopomers of CO.

In this work, we adopt the same basic model as in (Andric et al. 1999) to describe the molecular system and the non adiabatic coupling between the bound and dissociative states. However, to calculate the photodissociation cross section, we adopt an alternative approach (Andric et al. 2002). To perform the analytic continuation of the system's Green function into the complex energy plane (which is essential for a description of the resonances), the Hamiltonian is modified by the introduction of a smooth external complex scaling (SECS) (Moiseyev 1998). The SECS essentially consists in replacing the internuclear radial coordinate by a complex path whose direction is asymptotically defined by a "rotation angle" $\theta$.

To represent the state vectors and the modified Hamiltonian, we have chosen the discrete variable representation (DVR) corresponding to an equidistant set of radial grid points (Colbert \& Miller 1992). The cross-section calculations are performed by diagonalizing the (complex symmetric) Hamiltonian matrix and using a spectral expansion of the system's Green function. Those eigenvalues of the complex Hamiltonian which are stable with respect to variations of the rotation angle $\theta$ are designated resonant eigenvalues, as opposed to the non resonant eigenvalues, which are unstable with respect to variations of $\theta$. A more detailed discussion of this point is given in the following section.

\section{Method}

In our model (Andric et al. 1999), photodissociation occurs by an indirect process through absorption of a photon from the ground $\mathrm{X}^{1} \Sigma^{+}$state into a predissociating level of the excited $\mathrm{B}^{1} \Sigma^{+}$Rydberg state, which can dissociate via a strong non adiabatic coupling with the $\mathrm{D}^{\prime} \Sigma^{+}$repulsive valence state. However, the non adiabatic coupling is such that neither the bound nor the dissociative state can be adequately described by a single adiabatic (or diabatic) potential energy curve. In reality, it is necessary to treat the problem as an absorption from the ground state into a coupled pair of excited states. We have chosen to use a diabatic representation of the system as in (Andric et al. 1999), but it has been verified that identical results are obtained with an adiabatic representation.

A radial sinc-function discrete variable representation (DVR) basis (Colbert \& Miller 1992) is used to describe the state vectors and operators of the system on a set of $N_{\text {DVR }}$ equidistant grid-points: $R_{j}=R_{\min }+j \Delta$, $\Delta=\left(R_{\max }-R_{\min }\right) / N_{\mathrm{DVR}}, j=1,2, \ldots N_{\mathrm{DVR}}$. In this representation the matrix elements of all local operators (such as potentials and the diabatic couplings) are (to a good approximation) diagonal. On the other hand, the kinetic energy matrix elements are non-diagonal, but they can be expressed in a simple analytic form. An analytic continuation of the system's Green function into the complex energy plane is accomplished in the present calculations using the approach of the smooth exterior complex scaling (SECS), in which the internuclear radial coordinate is replaced by Moiseyev (1998):

$R \rightarrow R+(\exp (i \theta)-1)\left[R+\frac{1}{2 \lambda} \ln \left(\frac{\cosh \left[\lambda\left(R-R_{0}\right)\right]}{\cosh \left[\lambda\left(R+R_{0}\right)\right]}\right)\right]$

where $\theta$ is the rotation angle around the point of exterior rotation $\left(R=R_{0}\right)$ and $\lambda$ is the parameter defining the smoothness of the transformation.

The choice of the three parameters $\theta, R_{0}$ and $\lambda$ is governed by certain constraints. The angle $\theta$ must be sufficiently large so that the resonances are uncovered. The upper limit of $\theta$ is not very critical and in the present work, we have found the results to be stable for $\theta$ in the range $0.05 \leq \theta \leq 0.5$. The choice of $R_{0}$ is such that the potentials and the couplings have reached their asymptotic values. The results are stable for all $R_{0}$ satisfying this constraint. But since the computing time increases with $R_{0}$, in practice we choose the smallest possible value. The parameter $\lambda$ is chosen to be as large as possible consistent with the constraint of a smooth transformation from the region $\left(R<R_{0}\right)$ to the region $\left(R>R_{0}\right)$. A full discussion of the problem can be found in Moiseyev (1998). The results are stable over a wide range of $\lambda$.

The resulting complex symmetric radial Hamiltonian matrix $\mathbf{H}$ (Andric et al. 2002) representing the final coupled states is of dimension $2 N_{\mathrm{DVR}} \times 2 N_{\mathrm{DVR}}$. The expression for total photodissociation cross section, neglecting rotations $(J=0)$, (in atomic units) is given by (Andric et al. 2002):

$\sigma(\mathrm{E})=-\frac{4 \pi \omega}{3 \mathrm{c}} \operatorname{Im} G_{00}(E)$

where $\omega$ is the photon frequency and the Green function $G_{00}(\mathrm{E})$ is given by:

$G_{00}(E)=\boldsymbol{\Psi}_{0}^{T} .(E \mathbf{I}-\mathbf{H})^{-1} \cdot \boldsymbol{\Psi}_{0}$

and the $2 \times N_{\mathrm{DVR}}$ components of the vector $\boldsymbol{\Psi}_{0}$ are given by:

$\left(\Psi_{0}\right)_{\alpha, \mathrm{j}}=\sqrt{\Delta} \mathrm{D}_{0, \alpha}\left(R_{j}\right) \chi_{v_{0}}\left(R_{j}\right), \alpha=1,2 ; \mathrm{j}=1,2, \ldots ., N_{\mathrm{DVR}}$

where $\chi_{v_{0}}(R)$ is the initial vibrational wavefunction and $\mathrm{D}_{0, \alpha}(R)$ is the dipole transition moment between the initial $(0)$ and final $(\alpha)$ electronic states. To calculate the Green function (4) the 
most direct way is to diagonalize $\mathbf{H}$ in order to obtain the set of complex eigenvalues and eigenvectors:

$\left(E_{k}-\frac{i}{2} \Gamma_{k}, \boldsymbol{\Phi}_{k}\right), k=1,2, \ldots, 2 \times N_{\mathrm{DVR}}$.

These may then used to construct the spectral expansion:

$G_{00}(\mathrm{E})=\sum_{k=1}^{2 \times N_{\mathrm{DVR}}} \frac{\left(\boldsymbol{\Psi}_{0}^{T} \cdot \boldsymbol{\Phi}_{k}\right)^{2}}{E-E_{k}+\frac{\mathrm{i}}{2} \Gamma_{k}}$.

Note that in the above expression (7), the pseudo scalar product without the complex conjugation is used, as is appropriate for complex symmetric matrices.

In the present work, where the Hamiltonian matrix is fairly small (of the order of $500 \times 500$ ), a standard routine for complex symmetric matrices of the EISPACK package has been used to compute $E_{k}, \Gamma_{k}, \Phi_{k}$.

In our earlier work (Andric et al. 1999) on the normal $\mathrm{CO}$ isotope, the Green function was obtained by a recursive Chebyshev expansion of the Green operator. However, the convergence of the Chebyshev expansion was rather slow for the narrow resonance profiles. The spectral expansion (4) of the Green operator used in this work is remarkably fast and stable and is more satisfactory than the Chebyshev method for the calculation of photodissociation cross sections with narrow resonance features.

There is one other important point to bear in mind when using spectral expansions such as (4). The complex energy spectrum reveals two kinds of eigenvalues. The first kind of eigenvalues (designated as resonances) have an imaginary part, which is small in magnitude compared with the real part. These eigenvalues are stable with respect to variations of the parameters $\theta, R_{0}$ and $\lambda$. The second kind of eigenvalues, (designated as non-resonant), have real and imaginary components of comparable magnitude and are unstable with respect to variations of the parameters $\theta, R_{0}$ and $\lambda$. They contribute principally to the background continuum and interfere strongly with the resonance profiles. However, the spectral sum (4) is stable when a complete (or nearly complete) set of eigenvalues is used. This means that it is absolutely essential to include all eigenvalues of the transformed radial matrix $\mathbf{H}$ in the spectral sum. A more detailed discussion of this point may be found in Andric et al. (2002).

\section{Results}

Typical values of the parameters used in the cross section computations are the following. For the DVR grid: $R_{\min }=1.5 a_{0}$, $R_{\max }=5.3 a_{0}, N_{\mathrm{DVR}}=200$. For the smooth exterior complex scaling: $\theta=0.3, R_{0}=3.4 a_{0}, \lambda=10 a_{0}^{-1}$. The results of the cross section for photodissociation are presented graphically in Figs. 1-6. Figures 1, 3 and 5 cover the photon energy range from 90000 to $100000 \mathrm{~cm}^{-1}(11.15$ to $12.39 \mathrm{eV})$ and Figs. 2, 4 and 6 from 100000 to $106000 \mathrm{~cm}^{-1}$ (12.39 to $13.14 \mathrm{eV})$. Figures 1 and 2 present results for the isotopomers ${ }^{12} \mathrm{C}^{16} \mathrm{O},{ }^{12} \mathrm{C}^{17} \mathrm{O},{ }^{12} \mathrm{C}^{18} \mathrm{O}$, while in Figs. 3 and 4 the results for ${ }^{13} \mathrm{C}^{17} \mathrm{O},{ }^{13} \mathrm{C}^{18} \mathrm{O}$ are compared with those of ${ }^{12} \mathrm{C}^{16} \mathrm{O}$. In Figs. 5 and 6 the results for ${ }^{13} \mathrm{C}^{16} \mathrm{O}$ and ${ }^{12} \mathrm{C}^{18} \mathrm{O}$ are compared.

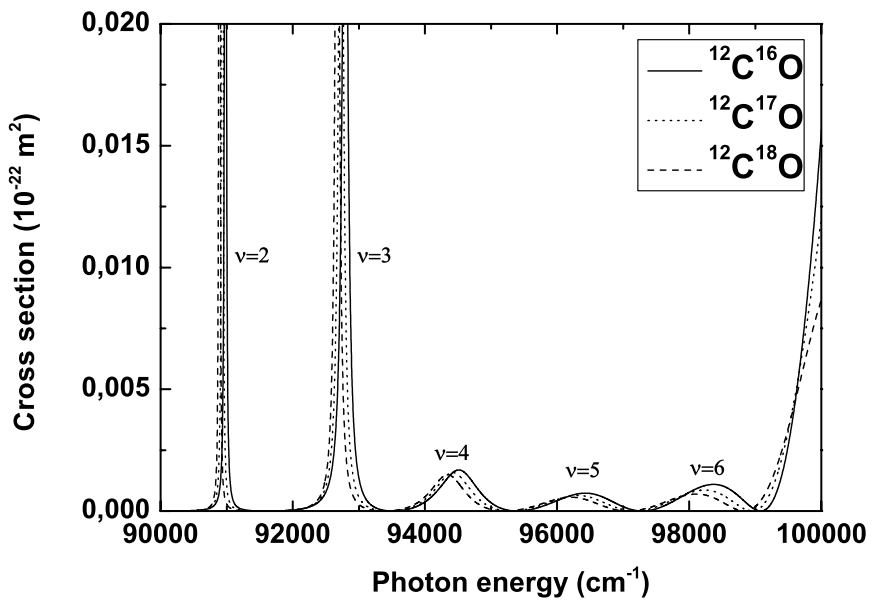

Fig. 1. Photodissociation cross section for the ${ }^{12} \mathrm{C}$-containing isotopes of $\mathrm{CO}$ in the photon energy range: $[90000,100000] \mathrm{cm}^{-1}$.

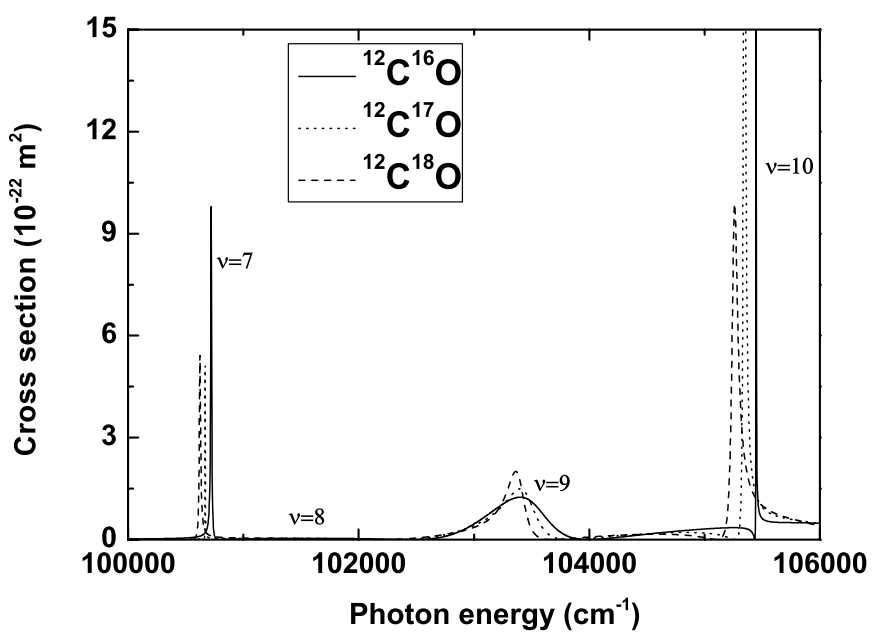

Fig. 2. Photodissociation cross section for the ${ }^{12} \mathrm{C}$-containing isotopes of $\mathrm{CO}$ in the photon energy range: $[100000,106000] \mathrm{cm}^{-1}$.

The cross sections are characterized by a series of resonance peaks, which may be labelled in ascending order by a number $v$. The lower resonances $(v=1-3)$ are shape resonances whose peak position corresponds to the vibrational states of the Rydberg state and whose width is directly related to the tunnelling probability through the adiabatic potential barrier. The width for these shape resonances, which is negligible for $v=0$, increases (as expected) with $v$, attaining a half width of about $30-40 \mathrm{~cm}^{-1}$ for $v=3$. It may be remarked that for $v \leq 3$ there is little overlap between the resonances of the different isotopomers.

The resonances $v \geq 4$, correspond to a coupled state system and cannot be identified with any vibrational state of a specific adiabatic (or diabatic) state. These resonances are typically of the Feshbach type. Their profiles have quite a complex structure arising from the interference between the resonances and the background continuum. Their most remarkable feature concerns their dependence on the reduced mass of the system. For the systems ${ }^{13} \mathrm{C}^{16} \mathrm{O}$ and ${ }^{12} \mathrm{C}^{18} \mathrm{O}$, whose reduced masses only differ by $0.3 \%$, there is an almost complete overlap of the cross section profiles throughout the spectral range. On the other 


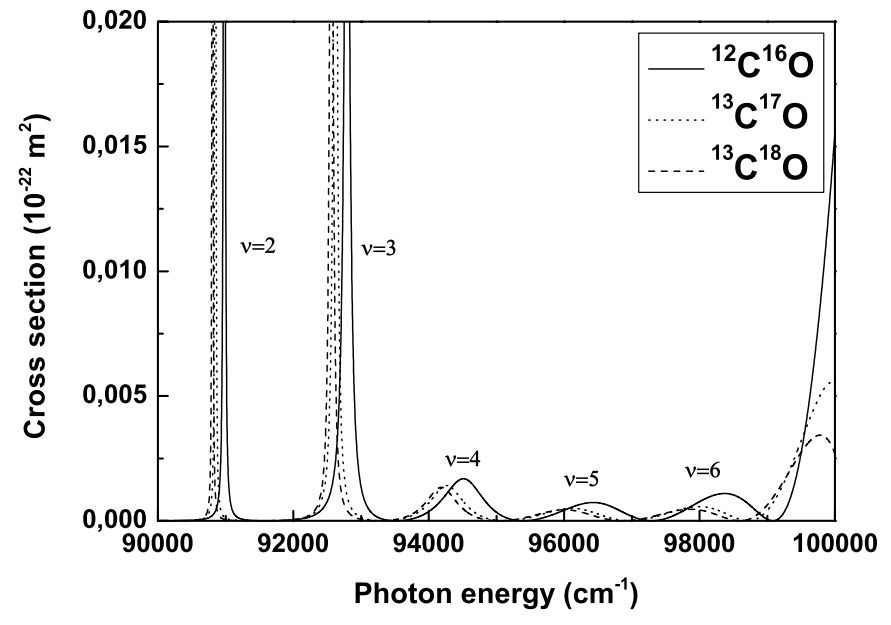

Fig. 3. Photodissociation cross section for the ${ }^{12} \mathrm{C}^{16} \mathrm{O}$, ${ }^{13} \mathrm{C}^{17} \mathrm{O}$ and ${ }^{13} \mathrm{C}^{18} \mathrm{O}$ isotopomers in the photon energy range: [90000, 100000$] \mathrm{cm}^{-1}$.

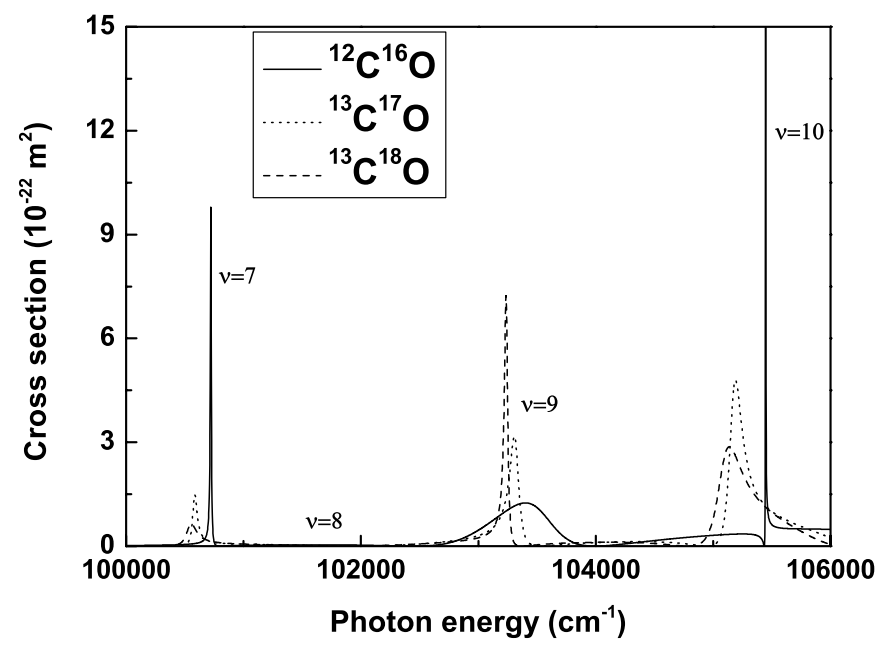

Fig. 4. Photodissociation cross section for the ${ }^{12} \mathrm{C}^{16} \mathrm{O}$, ${ }^{13} \mathrm{C}^{17} \mathrm{O}$ and ${ }^{13} \mathrm{C}^{18} \mathrm{O}$ isotopomers in the photon energy range: $[100000,106000] \mathrm{cm}^{-1}$.

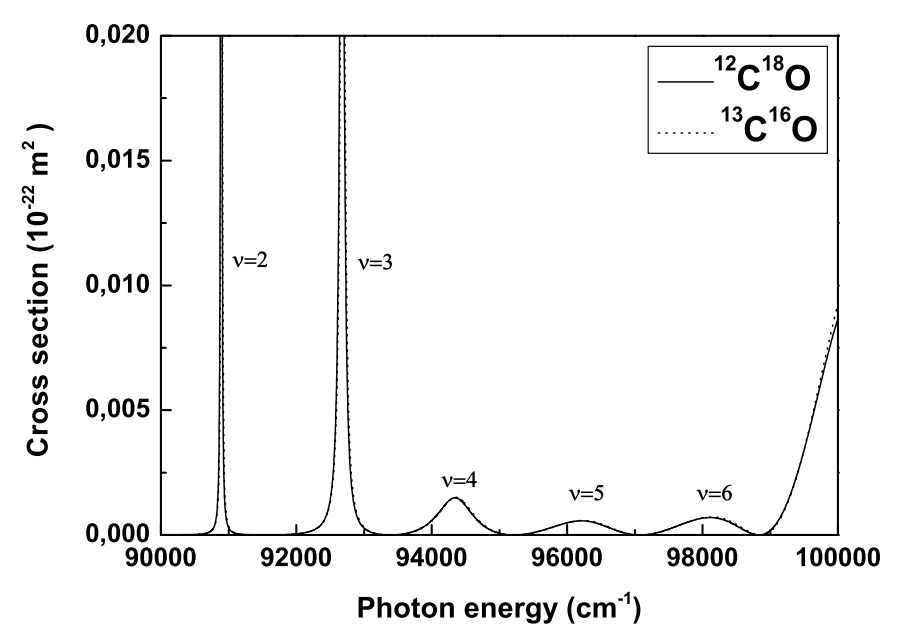

Fig. 5. Photodissociation cross section for the ${ }^{12} \mathrm{C}^{18} \mathrm{O}$, and ${ }^{13} \mathrm{C}^{16} \mathrm{O}$ isotopomers in the photon energy range: $[90000,100000] \mathrm{cm}^{-1}$.

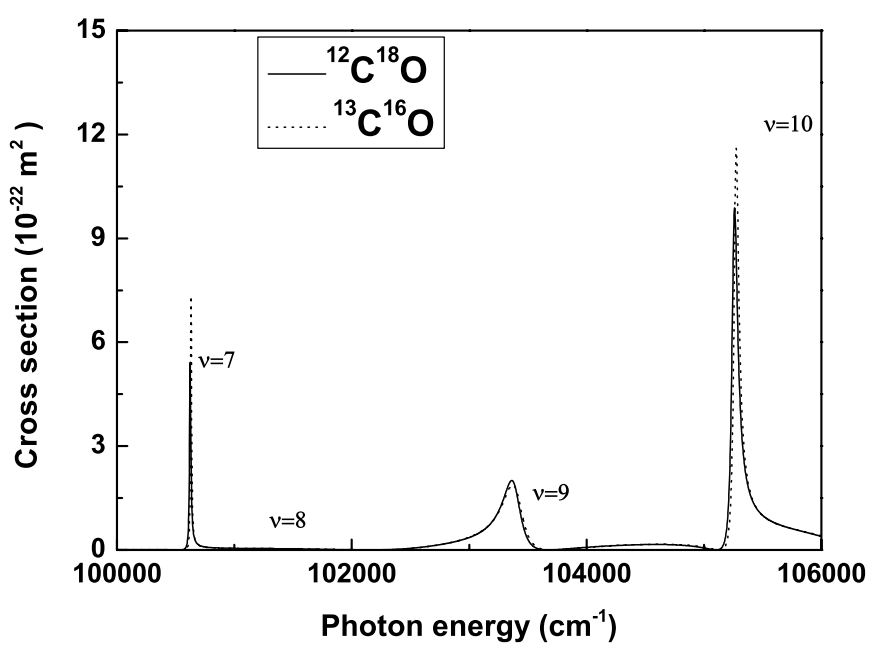

Fig. 6. Photodissociation cross section for the ${ }^{12} \mathrm{C}^{18} \mathrm{O}$, and ${ }^{13} \mathrm{C}^{16} \mathrm{O}$ isotopomers in the photon energy range: $[100000,106000] \mathrm{cm}^{-1}$.

hand for the other systems, the reduced mass differences are much greater (of the order of $2-5 \%$ ) and the cross section profiles vary quite markedly from one isotopomer to another. The weaker Feshbach resonances $(v=4,5,6,8)$ overlap completely while for the stronger resonances $(v=7,9,10)$, the overlap is considerable though not always complete.

Another important aspect of the present calculations can be deduced from Tables 1 and 2, where we tabulate the real and imaginary parts (respectively $E_{r}$ and $\Gamma_{r} / 2$ ) of the resonant eigenvalues of the complex Hamiltonian (the zero of the energy taken at the minimum of the diabatic $\mathrm{B}^{1} \Sigma^{+}$potential energy curve). The data for the normal isotopomer ${ }^{12} \mathrm{C}^{16} \mathrm{O}$ are in very good agreement with results obtained using alternative methods by Monnerville \& Robbe (1994), Li et al. (1997). The values of $E_{r}$ may be compared with $E_{\mathrm{p}}$, the positions of the calculated cross section peaks. The cross section widths are not tabulated as it is not always possible to assign a meaningful width to the cross section profiles corresponding to the Feshbach type resonances. It can be seen that, except for the weak resonances $(v=5,6,8)$ the real parts $E_{r}$ of the resonant eigenvalues correspond well to the positions of the cross section peaks. On the other hand, the value of $\Gamma_{r} / 2$ only gives a meaningful idea of the cross section profile for the low shape resonances $(v=1-3)$. For the Feshbach resonances $(v \geq 4)$ the values of $\Gamma_{r} / 2$ only follow qualitatively the trend of the changes of the cross section profiles. This underlines the remarks at the end of the preceding section on the necessity to retain in the spectral expansion (7) not only the contributions of the individual resonant eigenvalues, but also the contributions from all eigenvalues (both resonant and non resonant).

A data file with the numerical values of the computed cross sections is freely available to anyone interested.

\section{Conclusion}

In discussing the consequences of our calculations, it should, of course, be recognized that there are few direct experimental measurements of photo absorption experiments with which our 
Table 1. Values of $E_{r}, E_{\mathrm{p}}$ and $\Gamma_{r} / 2$ in $\mathrm{cm}^{-1}$ for the ${ }^{12} \mathrm{C}$-containing isotopes. Note that in this table, the zero of the energy scale is taken to be the minimum of the $\mathrm{B}^{1} \Sigma^{+}$potential energy curve.

\begin{tabular}{|c|c|c|c|c|c|c|c|c|c|}
\hline \multirow[t]{2}{*}{$v$} & \multicolumn{3}{|c|}{${ }^{12} \mathrm{C}^{16} \mathrm{O}$} & \multicolumn{3}{|c|}{${ }^{12} \mathrm{C}^{17} \mathrm{O}$} & \multicolumn{3}{|c|}{${ }^{12} \mathrm{C}^{18} \mathrm{O}$} \\
\hline & $E_{r}$ & $E_{\mathrm{p}}$ & $\Gamma_{r} / 2$ & $E_{r}$ & $E_{\mathrm{p}}$ & $\Gamma_{r} / 2$ & $E_{r}$ & $E_{\mathrm{p}}$ & $\Gamma_{r} / 2$ \\
\hline 0 & 905 & $\cdots$ & 0 & 892 & $\ldots$ & 0 & 880 & $\ldots$ & 0 \\
\hline 1 & 2988 & 2988 & $2 \times 10^{-4}$ & 2950 & 2950 & $14 \times 10^{-5}$ & 2915 & 2914 & $9 \times 10^{-5}$ \\
\hline 2 & 4971 & 4972 & .45 & 4911 & 4910 & 0.34 & 4856 & 4857 & .27 \\
\hline 3 & 6784 & 6784 & 45 & 6708 & 6708 & 38 & 6640 & 6639 & 33 \\
\hline 4 & 8514 & 8504 & 401 & 8407 & 8403 & 372 & 8311 & 8310 & 346 \\
\hline 5 & 10631 & 10421 & 927 & 10468 & 10292 & 897 & 10324 & 10178 & 869 \\
\hline 6 & 13235 & 12361 & 1067 & 13004 & 12210 & 1086 & 12800 & 12075 & 1095 \\
\hline 7 & 14713 & 14713 & 4.7 & 14646 & 14646 & 0.12 & 14589 & 14139 & 6.77 \\
\hline 8 & 16925 & 15597 & 595 & 16603 & $\ldots$ & 749 & 16313 & $\ldots$ & 823 \\
\hline 9 & 17534 & 17392 & 311 & 17438 & 17379 & 166 & 17354 & 17329 & 92 \\
\hline 10 & 19438 & 19438 & 0.14 & 19322 & 19323 & 9.06 & 19220 & 19226 & 30 \\
\hline
\end{tabular}

Table 2. Values of $E_{r}, E_{\mathrm{p}}$ and $\Gamma_{r} / 2$ in $\mathrm{cm}^{-1}$ for the ${ }^{13} \mathrm{C}$-containing isotopes. As in Table 1, the zero of the energy scale is taken to be the minimum of the $\mathrm{B}^{1} \Sigma^{+}$potential energy curve.

\begin{tabular}{cccccccccc}
\hline \hline$v$ & & ${ }^{13} \mathrm{C}^{16} \mathrm{O}$ & & \multicolumn{3}{c}{${ }^{13} \mathrm{C}^{17} \mathrm{O}$} & \multicolumn{2}{c}{${ }^{13} \mathrm{C}^{18} \mathrm{O}$} \\
& $E_{r}$ & $E_{\mathrm{p}}$ & $\Gamma_{r} / 2$ & $E_{r}$ & $E_{\mathrm{p}}$ & $\Gamma_{r} / 2$ & $E_{r}$ & $E_{\mathrm{p}}$ & $\Gamma_{r} / 2$ \\
\hline 0 & 882 & $\ldots$ & 0 & 868 & $\ldots$ & 0 & 856 & $\ldots$ & 0 \\
1 & 2920 & 2920 & $1 \times 10^{-4}$ & 2881 & 2880 & $7 \times 10^{-5}$ & 2845 & 2844 & $5 \times 10^{-5}$ \\
2 & 4865 & 4864 & 0.28 & 4803 & 4803 & 0.21 & 4746 & 4746 & 0.16 \\
3 & 6651 & 6651 & 34 & 6573 & 6573 & 28 & 6503 & 6503 & 24 \\
4 & 8328 & 8325 & 350 & 8220 & 8220 & 321 & 8125 & 8127 & 296 \\
5 & 10348 & 10197 & 874 & 10185 & 10066 & 841 & 10040 & 9947 & 811 \\
6 & 12834 & 12098 & 1094 & 12604 & 11945 & 1098 & 12399 & 11807 & 1095 \\
7 & 14598 & 14599 & 4.9 & 14536 & 14539 & 23 & 14486 & 14496 & 52 \\
8 & 16362 & $\ldots$ & 813 & 16032 & $\ldots$ & 863 & 15733 & $\ldots$ & 879 \\
9 & 17369 & 17339 & 103 & 17270 & 17261 & 47 & 17179 & 17177 & 17 \\
10 & 19237 & 19242 & 25 & 19124 & 19143 & 65 & 19029 & 19080 \\
\hline
\end{tabular}

results can be compared. Most laboratory experiments are carried out at room temperature, where many rotationally excited states of $\mathrm{CO}$ are present and it is difficult to extract with precision the fine details concerning the low rotational levels of interstellar interest. We shall therefore limit our discussion to the general features of our results, which do not depend critically on our assumptions concerning the non-adiabatic coupling matrix elements, but which have some relevance to the problem of isotopic fractionation in $\mathrm{CO}$.

Firstly, our results indicate that no differential selfshielding between ${ }^{13} \mathrm{C}^{16} \mathrm{O}$ and ${ }^{12} \mathrm{C}^{18} \mathrm{O}$ is expected throughout the entire spectral range from 90000 to $106000 \mathrm{~cm}^{-1}$. This implies that any overabundance of ${ }^{13} \mathrm{C}^{16} \mathrm{O}$ with respect to ${ }^{12} \mathrm{C}^{18} \mathrm{O}$ is due to an enrichment of ${ }^{13} \mathrm{C}^{16} \mathrm{O}$ by the collisional process (1). This conclusion is compatible with the observations of Penzias et al. (1972) and Casoli et al. (1992), which show no evidence for any differential shielding between these two isotopomers.
Secondly, we confirm that there is indeed a differential shielding between normal $\mathrm{CO}$ and the other isotopomers, but only for photon energies less than $94000 \mathrm{~cm}^{-1}$. However, for energies greater than $94000 \mathrm{~cm}^{-1}$, the photoabsorption profiles are wide (some as large as $1000 \mathrm{~cm}^{-1}$ ), and the absorption profiles of all the isotopomers overlap considerably. For that reason, we expect little differential shielding at energies greater than $94000 \mathrm{~cm}^{-1}$. To estimate of the global effect of differential shielding will require a knowledge of the distribution of the interstellar radiation intensity over the entire spectral range from 94000 to $106000 \mathrm{~cm}^{-1}$. An investigation of this problem is beyond the scope of this paper.

Acknowledgements. The authors acknowledge support of this work by the Centre National de la Recherche Scientifique (CNRS) in the framework of the Programme National Physico-Chimie Interstellaire. T.P.G. also acknoweledges support by the Ministry of Science, 
Technology and Development of the Republic of Serbia through the project No. 1470.

\section{References}

Andric, L., Grozdanov, T. P., McCarroll, R., \& Tchang-Brillet, W.-Ü.L. 1999, J. Phys. B: At. Mol. Opt. Phys., 32, 4729

Andric, L., Baccarelli, I., Grozdanov, T. P., \& McCarroll, R. 2002, Phys. Lett. A, 298, 41

Bensch, F., Pak, L., Wouterloot, J. G. A., Klapper, G., \& Winnewisser, G. 2001, ApJ, 562, L185

Boreiko, R. T., \& Betz, A. L. 1996, ApJ, 467, L113

Casoli, F., Dupraz, C., \& Combes, F. 1992, A\&A, 264, 49

Colbert, D. T., \& Miller, W. H. 1992, J. Chem. Phys., 96, 1982

Dickman, R. L., Langer, W. D., McCutcheon, W. H., \& Shuter, W. L. H. 1977, CNO Isotopes in Astrophysics, ed. J. Audouze (Dordrecht: Reidel), 95

Keene, J., Schilke, P., Kooi, J., et al. 1998, ApJ, 494, L107 (1998)
Langer, W. D., Goldsmith, P. F., Carlson, E. R., \& Wilson, R. W. 1980, ApJ, 235, L39

Li, Y., Bludski, O., Hirsch, G., \& Buenker, R. J. 1997, J. Chem. Phys., 107,3014

Mahoney, M. J., McCutcheon, W. H., \& Shuter, W. L. H. 1976, AJ, 81,508

Moiseyev, N. 1998, J. Phys. B: At. Mol. Opt. Phys., 31, 1431

Monnerville, M., \& Robbe, J. M. 1994, J. Chem. Phys., 101, 7580

Penzias, A. A., Jefferts, K. B., Wilson, R. W., Liszt, H. S., \& Solomon, P. M. 1972, ApJ, 178, L35

Sheffer, Y., Federman, S. R., Lambert, D. L., \& Cardelli, J. A. 1992, ApJ, 397, 482

Sheffer, Y., Lambert, D. L., \& Federman, S. R. 2002, ApJ, 574, L171

Smith, A. M., Bruhweiler, F. C., Lambert, D. L., et al. 1991, ApJ, 377, L61

van Dishoeck, E. F., \& Black, J. H. 1988, ApJ, 334, 771

Watson, W. D., Anicich, V. G., \& Huntress, W. T. 1976, ApJ, 205, L165 